\title{
Bone mineral density in patients with recent onset rheumatoid arthritis: influence of disease activity and functional capacity
}

R F J M Laan, W C A M Buijs, A L M Verbeek, M P Draad, F H M Corstens, L B A van de Putte, P L C M van Riel

\begin{abstract}
Background. Generalised osteoporosis is often described in patients with rheumatoid arthritis (RA). The aim of this study was to evaluate disease related determinants of bone mineral density (BMD) in patients with RA. Methods. Subjects were selected from a group of 147 patients with recent onset RA. Disease activity and functional capacity were studied prospectively in this cohort. Activity of the disease was assessed once every three months by various parameters, and functional capacity was measured with a health assessment questionnaire once every six months. Ninety seven patients consented to participate in the study. Bone mineral density was assessed with dual energy $x$ ray absorptiometry in the lumbar spine, in a combined region of interest in the hips, and in Ward's triangle. Multiple linear regression procedures were used to analyse the data.
\end{abstract}

Results. Duration of RA was negatively associated with BMD at all three sites of measurement. The mean erythrocyte sedimentation rate in the six months before BMD measurement was negatively associated with BMD in the hip and in Ward's triangle. Other parameters of disease activity were not related to BMD. The mean health assessment questionnaire score in the 18 months before BMD measurement was negatively associated with $B M D$ in the combined hip region only. Bone mineral density tended to be decreased when patients were compared with a normal reference group, especially in the femoral regions of interest.

Conclusions. It is concluded that BMD may be affected in patients with recent onset $R A$ by disease dependent mechanisms. Several factors have been suggested elsewhere as determinants of BMD in RA. The results of this study show that disease duration, disease activity, and functional impairment may, independently of each other, contribute to bone loss, especially in the proximal femur.

(Ann Rheum Dis 1993; 52: 21-26)

The pathogenesis of axial osteopenia in patients with rheumatoid arthritis (RA) is poorly understood. As in patients without RA, age, sex, height, weight, parity, time since the menopause, drinking and smoking history, and some dietary habits may affect bone mineral density (BMD). ${ }^{1}$ In addition, several factors specifically related to RA could also be important. Most often the duration of the disease, the degree of functional impairment, and the severity of the inflammatory process have been suggested as potential risk factors for osteoporosis in patients with RA.

Most previous studies have had a cross sectional design. A negative association between duration of RA and BMD was found in some of these studies, ${ }^{2}{ }^{3}$ but has not been confirmed by others. ${ }^{4-8}$ The degree of functional impairment has been sugested as an important risk factor for fracture by some workers, ${ }^{910}$ but not by all. ${ }^{11} \mathrm{~A}$ negative association between the latter factor and BMD has been described, ${ }^{2}{ }^{7}$ and denied. ${ }^{468}$ Disease activity has also been suggested as a possible risk factor for osteopenia in patients with rheumatoid arthritis. ${ }^{34}$ Other workers found no such association. ${ }^{12}$

As many, often interdependent, factors may contribute to the development of osteoporosis in patients with RA, the influence of one specific factor is difficult to evaluate, and the effect of potentially confounding factors on the results should be corrected for. So far, no study has included all the suggested disease related risk factors in an analysis suitable for the evaluation of the influence of multiple factors on BMD. Furthermore, these studies have only assessed potential risk factors such as degree of functional impairment and activity of RA at the time when the BMD was measured. It may be expected, however, that their effects will only become apparent after a sufficiently long period of time. Therefore these data should be interpreted with restraint. In our department, disease activity and functional capacity are studied prospectively in all patients with recent onset RA. These data were used to study the influence of $R A$ related factors on axial BMD.

Patients and methods

PATIENTS

Since 1985 all patients presenting to the rheumatology outpatient clinic of the University Hospital Nijmegen with recent onset RA have been asked to participate in a long term prospective follow up study. All patients included in this study had classical or definite $\mathrm{RA},{ }^{13}$ had had symptoms for less than one year, and had not been treated with disease modifying antirheumatic drugs before inclusion in the study. At the start of the present investigation 147 patients were participating in the long term prospective follow up. All patients were asked to participate in the present study. 
prospectively assessed in all participating patients by specially trained research nurses. Measures of disease activity were assessed at least once every three months. For the present study only three monthly assessments were used. Functional capacity was assessed once every six months.

Measures of disease activity included the number of tender joints, the number of swollen joints, the Ritchie articular index,${ }^{14}$ duration of morning stiffness (minutes), severity of pain (100 $\mathrm{mm}$ visual analogue scale: 0 indicating no and 100 indicating maximum pain), degree of general health $(100 \mathrm{~mm}$ visual analogue scale: 0 indicating best and 100 indicating worst possible), left and right hand grip strength with a vigorimeter $(\mathrm{mmHg})$, erythrocyte sedimentation rate (ESR) according to Westergren ( $\mathrm{mm} /$ first hour), haemoglobin (g/l), thrombocyte count $\left(10^{9} / \mathrm{l}\right)$, total protein, albumin, $\alpha_{1}$ globulin, $\alpha_{2}$ globulin, $\beta$ globulin, and $\gamma$ globulin (all $\mathrm{g} / \mathrm{l}), \mathrm{C}$ reactive protein $(\mathrm{g} / \mathrm{l})$, and $\operatorname{IgM}$ rheumatoid factor (IU/ml).

Functional capacity was assessed by a Dutch equivalent of the Stanford Health Assessment Questionnaire (range from 0.00 to 3.00 ; a zero score indicates no disability) that was validated in our department for patients with RA. ${ }^{15}$

At each visit the use of any drugs, including glucocorticoids, was noted.

\section{OTHER CLINICAL MEASURES}

At the time of bone mineral densitometry additional information was collected on some clinical variables. Height and weight were determined and a body mass index, expressed in $\mathrm{kg} / \mathrm{m}^{2}$, was calculated in all patients. In women the menopausal state, the time since the menopause (years), and the number of children were also noted.

\section{BONE MINERAL DENSITOMETRY}

Bone mineral density was measured once by dual energy $x$ ray absorptiometry with a Hologic QDR 1000 (Hologic Inc, Waltham, MA, USA) instrument at the lumbar spine (L1-L4) and at the left and right hips. The results of the two hip measurements were averaged. In some patients BMD could not be assessed in one or both hips because of hip replacement. Bone mineral density in the hips was assessed in a combined region including the femoral neck, the trochanteric and intertrochanteric region, and also separately in Ward's triangle, a region with a greater proportion of trabecular bone that may be important for femoral bone strength. ${ }^{16}$ The dual energy $x$ ray absorptiometry results are expressed in $\mathrm{g} / \mathrm{cm}^{2}$, and in $Z$ scores (number of standard deviations above or below the normal mean after comparison with age and sex matched normal control values, based on measurements in a North American population supplied by the manufacturer). In vivo reproducibility was evaluated in 60 women (mean age 51.4 years). The coefficient of variation was $1.0 \%$ for the lumbar spine, $1.7 \%$ for the combined region in the hip, and $2 \cdot 4 \%$ for Ward's triangle.
ANALYTICAL AND STATISTICAL METHODS

The duration of RA was defined as the duration of follow up plus the duration of symptoms before entry in the prospective study, and was expressed in months.

Disease activity was evaluated prospectively by different parameters as described earlier. In previous reports on the prospective follow up study, the Ritchie articular index, the number of swollen joints, the ESR, and the degree of general health were found to best reflect different aspects of disease activity. A combined disease activity score, consisting of those parameters, was also constructed. ${ }^{17}{ }^{18}$ In the present study we limited the analysis to the disease activity score and its four constituents. We first inspected plots between BMD and each of the variables reflecting disease activity. Initially, only those assessments carried out at approximately the same time as densitometry were used. We then repeatedly extended backwards the observation period with three month intervals, plotting the mean value of the disease activity score and each of the four constituting parameters against BMD, and the observation period with the best linear association between disease activity and BMD was selected.

A similar procedure was carried out for defining functional capacity. We plotted the health assessment questionnaire scores against $B M D$, and used six instead of three month intervals for the backwards extension of the observation period.

The influence of duration of RA, disease activity, and functional capacity on BMD was then evaluated by multiple linear regression analysis. The results obtained with dual energy $x$ ray absorptiometry in the lumbar spine, the combined region in the hip, and in Ward's triangle were used as dependent variables. The independent variables included age (years), gender $(0=$ male, $1=$ female $)$, body mass index $\left(\mathrm{kg} / \mathrm{m}^{2}\right)$, duration of RA (months), and disease activity and functional capacity as defined according to the procedures described above. In a subgroup of female patients the duration of time since the menopause (years) and parity (number of children) were also included among the independent variables. The regression analysis was carried out both with and without model selection techniques. Model selection was carried out by stepwise regression. In this analysis variables were added one by one to the model if they contributed to the BMD with $p \leqslant 0 \cdot 15$. After adding a variable, all variables already included in the model were re-evaluated and deleted if they no longer contributed to the BMD with $p \leqslant 0 \cdot 15$. We also checked whether the models finally selected best explained the variation in BMD by calculating $R^{2}$ (adjusted for the number of variables) for all possible models.

The relation between $\mathrm{BMD}$ on the one hand, and the various independent variables on the other, is described in three regression equations for each skeletal region separately. The proportion of variance of BMD explained by the regression equation is indicated by $R^{2}$, which has also been calculated after correction for the number of variables in the equation 


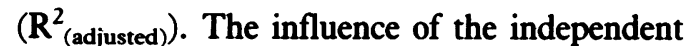
variables selected for the model on BMD is described in further detail by providing the parameter estimate, its $95 \%$ confidence interval, and the probability for testing the null hypothesis that the parameter estimate is not significantly different from zero. To facilitate the comparison between independent variables measured in different units, standardised parameter estimates have also been calculated. Differences in parameter estimates between subgroups of patients were evaluated with the $t$ test. All statistical analyses were carried out with the SAS/STAT software package. ${ }^{19}$

\section{Results}

At the time of the present study 147 patients with recent onset $R A$ participated in the prospective follow up for at least three months. A total of 97 patients agreed to bone mineral densitometry. The 50 patients who refused to undergo bone densitometry did not differ from the participants with respect to age, sex, or duration and severity of RA. Table 1 gives

Table 1 Clinical data. Results given as median (range)

\begin{tabular}{lll}
\hline Clinical parameter & Women $(n=58)$ & Men $(n=39)$ \\
\hline Age (years) & $57 \cdot 5$ & 57 \\
Number postmenopausal & $(27-78)$ & $(30-80)$ \\
Time since the menopause (years) & 41 & Not applicable \\
Parity (number of children) & 12 & Not applicable \\
& $(1-32)$ & \\
Body mass index (kg/m $\left.{ }^{2}\right)$ & $(0-9)$ & $24 \cdot 9$ \\
& $24 \cdot 0$ & $(20 \cdot 9-30 \cdot 0)$ \\
Duration of RA (months) & $(17 \cdot 6-34 \cdot 8)$ & 32 \\
& $30 \cdot 5$ & $(3-53)$ \\
Mean erythrocyte sedimentation rate (last & $(5-65)$ & 15 \\
six months; mm/first hour) & 22 & $(2-54)$ \\
Mean functional capacity (last 18 months, & $0 \cdot 28$ & $0 \cdot 25$ \\
HAQ $Q^{*}$ score 0-3) & $(0-2 \cdot 44)$ & $(0-1 \cdot 72)$ \\
Number receiving prednisone & 17 & 5
\end{tabular}

${ }^{*} \mathrm{HAQ}=$ health assessment questionnaire.

Table 2 Bone mineral density (BMD). Results given as mean (95\% confidence interval). Bone mineral density is measured in the lumbar spine (L1-L4), the hip (average of the two sides, combined region of interest including femoral neck, trochanteric, and intertrochanteric region), and in Ward's triangle (average of both sides)

\begin{tabular}{lll}
\hline & Women & Men \\
\hline Lumbar spine & $\mathrm{n}=58$ & $\mathrm{n}=39$ \\
$\mathrm{BMD}\left(\mathrm{g} / \mathrm{cm}^{2}\right)$ & 0.952 & 0.953 \\
$Z$ score & $(0.907$ to 0.997$)$ & $(0.906$ to 1.000$)$ \\
Hip & +0.33 to & -0.67 \\
BMD $\left(\mathrm{g} / \mathrm{cm}^{2}\right)$ & $(-0.06$ to +0.72$)$ & $(-1.13$ to -0.22$)$ \\
& $\mathrm{n}=52$ & $\mathrm{n}=39$ \\
$Z$ score & 0.821 & 0.906 \\
Ward's triangle & $(0.781$ to 0.860$)$ & $(0.866$ to 0.946$)$ \\
BMD $\left(\mathrm{g} / \mathrm{m}^{2}\right)$ & -0.27 & -0.33 \\
$Z$ score & $(-0.57$ to +0.04$)$ & $(-0.59$ to -0.06$)$ \\
& $\mathrm{n}=52$ & $\mathrm{n}=39$ \\
& 0.527 & 0.535 \\
& $(0.481$ to 0.573$)$ & $(0.497$ to 0.573$)$ \\
& -0.55 & -0.73 \\
& $(-0.87$ to -0.22$)$ & $(-0.99$ to -0.47$)$ \\
\hline
\end{tabular}

Table 3 Proportion of patients with low bone mineral density (BMD)

\begin{tabular}{|c|c|c|c|c|c|}
\hline \multirow[t]{2}{*}{$Z$ score } & \multirow[t]{2}{*}{$\begin{array}{l}\text { Expected } \\
\text { proportion }(\%)^{*}\end{array}$} & \multicolumn{4}{|c|}{$\begin{array}{l}\text { Proportion (\%) of patients ( } 95 \% \text { confidence } \\
\text { interval) with } B M D \leqslant Z \text { score }\end{array}$} \\
\hline & & Lumbar spine & Hip & & Ward's triangle \\
\hline $\begin{array}{l}-1.0 \\
-1.645 \\
-2.0\end{array}$ & $\begin{array}{r}15 \cdot 9 \\
5 \cdot 0 \\
2 \cdot 3\end{array}$ & $\begin{aligned} 32 \cdot 0 & (22 \cdot 7 \text { to } 41 \cdot 2) \\
15.5 & (8 \cdot 3 \text { to } 22 \cdot 7) \\
9.3 & (3.5 \text { to } 15 \cdot 0)\end{aligned}$ & $\begin{array}{r}24 \cdot 2 \\
11 \cdot 0 \\
4 \cdot 4\end{array}$ & $\begin{array}{l}(15 \cdot 4 \text { to } 33 \cdot 0) \\
(4 \cdot 6 \text { to } 17 \cdot 4) \\
(0.2 \text { to } 8 \cdot 6)\end{array}$ & $\begin{aligned} 39 \cdot 6 & (29 \cdot 5 \text { to } 49 \cdot 6) \\
13 \cdot 2 & (6 \cdot 2 \text { to } 20 \cdot 1) \\
6.6 & (1.5 \text { to } 11 \cdot 7)\end{aligned}$ \\
\hline
\end{tabular}

* Expected proportion of subjects with a BMD $\leqslant Z$ score (first row) in the study group (assuming a normal distribution). detailed clinical data on the participating patients. In those patients receiving prednisone the daily dose never exceeded $10 \mathrm{mg} / \mathrm{day}$. The median duration of glucocorticoid treatment was $1 \cdot 4$ years (range $0 \cdot 2-2 \cdot 7$ years).

Tables 2 and 3 summarise the BMD data. Lumbar BMD was measured in all 97 patients. In six patients no measurements could be performed in the hips because of bilateral joint replacement. $Z$ scores tended to be negative in our patients, indicating a decrease in mean BMD compared with the data of a normal age and sex matched reference population supplied by the manufacturer. The mean $Z$ score was positive only for the lumbar spine in female patients. The proportion of patients with a low BMD (table 3) was consistently higher than expected.

In the plots between $\mathrm{BMD}$ and the parameters reflecting disease activity, no association was seen for any of the observation periods between the BMD and Ritchie articular index, number of swollen joints, degree of general health, or the combined disease activity score. A negative linear association was suggested between the ESR and BMD for simultaneous measurements. When extending the observation period backwards, the association between ESR and BMD improved and was judged to be optimum for a period of six months preceding bone mineral densitometry. Gradual worsening of the observed association resulted from extending the observation period further backwards. For the purpose of the present study disease activity was then defined as the mean ESR in the six months before bone mineral densitometry (mean $\mathrm{ESR}_{6}$ ).

In the plots between BMD and health assessment questionnaire scores a linear association was suggested for simultaneous assessments. The association was judged to be optimum for an observation period of 18 months before densitometry. For the purpose of the present study functional capacity was then defined as the mean health assessment questionnaire score in the 18 months preceding BMD measurement (mean $\mathrm{HAQ}_{18}$ ).

Multiple linear regression analysis was carried out first using the data of all patients together. The following models were reached with the stepwise regression analysis:

$$
\begin{aligned}
& \text { BMD }_{\text {lumbar spine }}=0.911-\left(10^{-3} \times 2.94 \times \text { age }\right)+\left(10^{-2} \times 1.05 \times \mathrm{BMI}\right) \\
& -\left(10^{-3} \times 1.87 \times \text { duration } R A\right) \\
& \mathbf{R}^{2}=0 \cdot 13 ; \mathbf{R}_{(\text {adjusted) }}^{2}=0 \cdot 10 ; p=0.004 \\
& R^{2}=0.42 ; R^{2}
\end{aligned}
$$

(where $\mathrm{a}=0$ if male and $\mathrm{a}=1$ if female)

$\mathrm{BMD}_{\text {ward's Iriangle }}=0.779-\left(10^{-3} \times 4.62 \times\right.$ age $)+\left(10^{-2} \times 0.66 \times\right.$ BMI $)-\left(10^{-3} \times 2 \cdot 81 \times\right.$ duration RA $)$

$-\left(10^{-3} \times 2.46 \times\right.$ mean ESR $\left._{6}\right)-\left(10^{-2} \times 3.73 \times\right.$ mean $\left.\mathrm{HAQ}_{18}\right)$ $R^{2}=0.47 ; R^{2}{ }_{\text {(adjusted })}=0.42 ; p=0.0001$

Table 4 gives detailed information on the parameter estimates.

When $Z$ scores were used as the dependent variable, similar results were reached with respect to the influence of duration of RA, mean $\mathrm{ESR}_{6}$, and mean $\mathrm{HAQ}_{18}$ 
Table 4 Influence of various factors on bone mineral density in different skeletal regions: results of stepwise multiple linear regression analysis

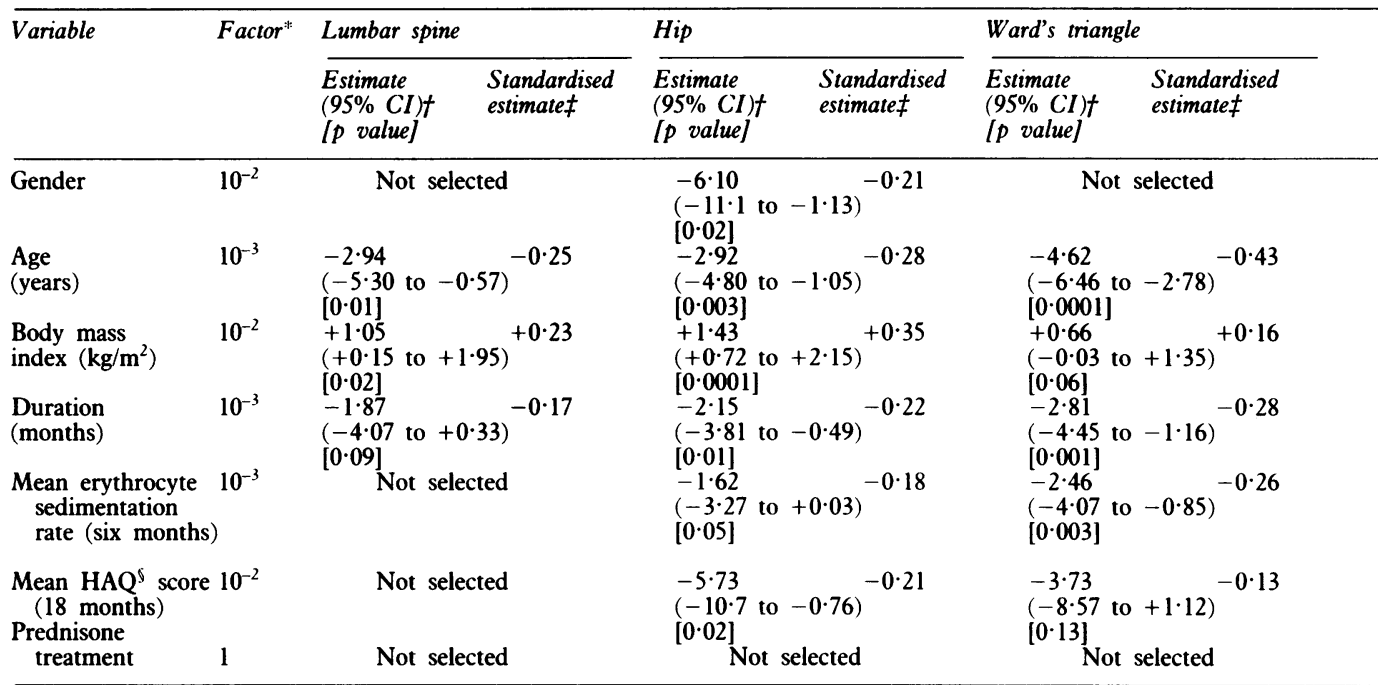

${ }^{*}$ Factor by which stated parameter estimate must be multiplied.

tCI=confidence interval.

\$Standardised estimates are calculated by dividing the parameter estimate by the ratio of the standard deviation of the bone minera density variable to the standard deviation of the parameter estimate.

$\$ H A Q=$ health assessment questionnaire.

In an attempt to exclude patients with false increases in lumbar BMD due to severe degenerative spinal changes, we checked the images produced by the Hologic bone densitometer for the presence of osteophytes. Patients who had less than two vertebrae with marked osteophytes were analysed separately. The mean $Z$ score in the lumbar spine was $-0 \cdot 19$ ( $95 \%$ confidence interval from -0.59 to +0.23 ) in the remaining 41 female patients, and -1.05 ( $95 \%$ confidence interval from -1.45 to -0.65 ) in the remaining 31 male patients. With the stepwise regression analysis the following model was reached

$\mathrm{BMD}_{\text {l.umbar spine }}=1.027-\left(10^{-3} \times 4.36 \times\right.$ age $)+\left(10^{-2} \times 0.84 \times \mathrm{BMI}\right)$ $-\left(10^{-3} \times 2 \cdot 80 \times\right.$ duration $\left.\mathrm{RA}\right)$

$$
\mathrm{R}^{2}=0 \cdot 28 ; \mathrm{R}_{(\text {adiusted })}^{2}=0 \cdot 25 ; \mathrm{p}=0.0001
$$

The $95 \%$ confidence intervals for the parameter estimates were -6.68 to -2.05 for age $(p=0.003),-4.89$ to -0.71 for duration of $R A$ $(p=0.009)$, and -0.07 to +1.76 for body mass index $(p=0.07)$

Further analysis showed that the models resulting from the stepwise regression analysis were also those with the highest $R^{2}$ value, after adjustment for the number of variables included.

Multiple linear regression analysis was also performed in subgroups of premenopausal and postmenopausal women, and in male patients. The parameter estimates for disease duration, $\mathrm{ESR}_{6}, \mathrm{HAQ}_{18}$, and body mass index were almost identical and not significantly different between those three subgroups. Prednisone treatment affected BMD in the combined region of the hip only in postmenopausal women (estimated influence of prednisone treatment $-0.088,95 \%$ confidence interval from -0.167 to $-0.008 ; p=0.03$ ). The age related decrease in BMD was significantly greater in postmenopausal women than in premenopausal women or in male patients, both in the lumbar spine and in the hip areas ( $p<0.01$ for all skeletal regions).
In postmenopausal women a separate analysis was carried out including time since the menopause and parity among the independent variables. Age and time since the menopause were highly correlated. Time since the menopause was included instead of age in the mode for the lumbar spine and for the combined region in the hip. Age was selected for the model for Ward's triangle, and time since the menopause was not included. Parity was included only in the model for lumbar BMD (parameter estimate $+0.022,95 \%$ confidence interval from -0.003 to $+0.048 ; p=0.09$ ). The inclusion of time since the menopause and parity among the independent variables in the analysis did not significantly change the estimates for duration of RA, mean $\mathrm{ESR}_{6}$, mean $H A Q_{18}$, use of prednisone, and body mass index.

\section{Discussion}

This study was designed to evaluate the influence of certain disease characteristics on axial bone mass in RA. For this purpose we used prospectively assessed measures of functional impairment and of activity of the inflammatory process, and we measured BMD in the lumbar spine and in the hips of patients with recent onset RA.

A negative association between duration of RA and BMD was found in three skeletal regions. This has been described by some, but not all, previous workers. ${ }^{2-8}$ Our data suggest that RA dependent mechanisms cause a gradual decrease in bone mass within the first five years of the disease in the axial skeleton.

The degree of physical activity is generally recognised as a determinant of BMD. Extreme inactivity causes rapid bone loss, whereas athletes have a higher BMD than sedentary subjects. Exercise intervention programmes have shown reduced bone loss or increased bone 
mass. ${ }^{20} 21$ In patients with RA impaired functional capacity and reduced physical activity have been associated with a decrease in bone mass and an increase in fracture risk in some cross sectional studies. ${ }^{27910}$ None of these studies included prospective data on functional capacity or disease activity. Our data show that the degree of functional impairment, expressed by the mean value of the health assessment questionnaire score in the last 18 months, is related to BMD in the hip areas, even after correction for differences in ESR.

The level of activity of the inflammatory process has also been suggested as a determinant of BMD in patients with RA in some cross sectional studies. ${ }^{34}$ Our data show that some but not all measures of disease activity may be related to femoral BMD. The mean ESR in the last six months was negatively associated with BMD in the hip areas. This association was no longer present when the observation period was extended over six months before densitometry, suggesting that only recent disease activity is important for BMD. Other measures of disease activity, including the Ritchie articular index, the number of swollen joints, and the degree of general health, were not related to BMD. The data suggest that the acute phase response may be associated with a release of factors that affect bone metabolism. Probable candidates are cytokines such as interleukin 1 and tumour necrosis factor, which are probably involved in the pathogenesis of $\mathrm{RA} .^{22}$ These factors are known to influence bone metabolism, ${ }^{23}$ and interleukin 1 has been suggested as a pathogenetic factor in patients with high bone turnover osteoporosis. ${ }^{24}$

The proportion of variance of lumbar BMD explained by the regression equation is low, and sex, mean ESR, and mean health assessment questionnaire score were not related to spinal BMD in our patients. We suspect that this may be due to measurement errors in some of our patients. False increases in lumbar BMD may result from vertebral fractures, from aortic calcifications, or from degenerative spinal changes. As no spinal radiographs were obtained, these possible explanations cannot be properly evaluated. However, checking the BMD of individual vertebrae did not suggest the presence of lumbar vertebral fractures in our patients. We also attempted to correct for the influence of possible degenerative spinal changes by checking the images produced by the densitometer for marked osteophytes. Stepwise regression analysis in the resulting subgroup showed an approximately twofold improvement of $\mathbf{R}^{2}$ for the lumbar spine. Sex, mean ESR, and mean health assessment questionnaire score, however, still had no effect on spinal BMD. We conclude that our lumbar BMD data should be cautiously interpreted.

The regression analysis was performed in the two sexes combined. No significant differences were observed in the estimated influence of duration of $R A$, degree of functional impairment, or level of disease activity, between the sexes or between pre- and postmenopausal women. The number of patients in each subgroup is small, however, and differences cannot actually be excluded. In postmenopausal women multiparity exerted a protective effect on BMD in the spine, as was previously described by Sambrook et al. ${ }^{7}$

No effect of prednisone on BMD could be shown except in postmenopausal women. Data on the influence of low dose glucocorticoids on bone mass in patients with RA are controversial. ${ }^{25}$ The present study was not designed to evaluate the possible contribution of this drug to the development of osteoporosis, and the number of patients using prednisone in the study is too small to allow conclusions on this topic. The data presented in this paper support the hypothesis that BMD may be affected by $\mathrm{RA}$ related mechanisms. This may lead to a decreased BMD in patients with $R A$ versus normal controls. We cannot, however, definitely confirm this hypothesis, as our study did not include normal controls. Instead, $Z$ scores were calculated after comparison with reference values supplied by the manufacturer. For this data set measurements were carried out in 950 (spine) and 1500 (hip) North American subjects. Reference data accumulated in France, Belgium, and Australia correlate closely with these North American values, within $\pm 2 \%$ over the whole BMD range for spinal measurements. ${ }^{26}$ Furthermore, 60 healthy women who participated in a trial aimed at the prevention of postmenopausal bone loss were measured in our hospital. Baseline measurements in these patients confirm the validity of the Hologic normal data for Dutch women aged between 40 and 60 . Mean $Z$ scores in our study were negative with the exception of the $Z$ score for the lumbar spine in female patients. After exclusion of patients with suspected severe degenerative spinal changes, $Z$ scores were also negative in this subgroup. The proportion of patients with a low bone mass was consistently higher in our patients than expected. Taken together our data suggest that BMD may be decreased in a subset of patients with recent onset RA, with active disease and functional disabilities.

Although clinical parameters were collected prospectively in our study, BMD was measured only once. Sambrook et al ${ }^{27}$ have previously prospectively studied a small number of patients with recent onset RA. Over a period of one to two years increased bone loss was shown at the distal radius, but not at the proximal radius or the lumbar spine. The rate of lumbar bone loss was, however, positively correlated with the serum concentrations of $C$ reactive protein. A preliminary report of a prospective study of BMD in recent onset RA, involving a greater number of patients, has been published by Gough et $a l,{ }^{28}$ and our results are in accordance with their findings; patients with active disease were at increased risk of spinal bone loss, whereas patients with greater functional impairment had a higher risk for femoral bone loss.

Previous reports on bone mass in patients with RA have shown contradictory results. ${ }^{27}$ In this study the separate influences of disease duration, disease activity, and functional impairment on BMD were evaluated. We 
conclude that BMD may be affected by RA dependent mechanisms, and that functional impairment and disease activity may be determinants of bone loss in patients with recent onset RA.

The authors thank E Brummelkamp for his assistance in analysing the data. This research was supported by a grant from the Dutch Ministry for Education (programme for the stimulation of health research, 'SGO').

1 Cummings S R, Kelsey J L, Nevitt M C, O’Dowd K J. Epidemiology of osteoporosis and osteoporotic fractures. Epidemiology of osteoporosis and

2 Als O S, Gotfredsen A, Riis B, Christiansen C. Are disease duration and degree of functional impairment determinants of bone loss in rheumatoid arthritis? Ann Rheum Dis 1985; 44: 406-11.

3 Reid D M, Kennedy N S, Smith M A, Tothill P, Nuki G. Total body calcium in rheumatoid arthritis: effects of disease activity and corticosteroid treatment. BMF 1982; 285: $330-2$.

4 Oka M, Rekonen A, Kuikka J, Anttinen J. Bone mineral density in rheumatoid arthritis measured by the gamma transmission method.

5 Nagant de Deuxchaisnes C, Devogelaer J P, Esselinckx W, et al. The effect of low dosage glucocorticoids on bone mass in rheumatoid arthritis: a cross-sectional and a longitudinal study using single photon absorptiometry. Adv Exp Med Biol 1984; 171: 209-39.

6 O'Malley M, Kenrick A J, Sartoris D J, et al. Axial bone density in rheumatoid arthritis: comparison of dual-energy projection radiography and dual-photon absorptiometry. projection radiography and ${ }_{\text {Radiology } 1989 ; 170: 501-5 \text {. }}$.

7 Sambrook P N, Eisman J A, Champion G D, Yeates M G, Pocock N A, Eberl S. Determinants of axial bone loss in rheumatoid arthritis. Arthritis Rheum 1987; 30: 721-8.

8 Compston J E, Crawley E O, Evans C, O'Sullivan M M. Spinal trabecular bone mineral content in patients with non-steroid treated rheumatoid arthritis. Ann Rheum Dis 1988; 47: $660-4$.

9 Hooyman J R, Melton III L J, Nelson A M, O'Fallon W M, Riggs B L. Fractures after rheumatoid arthritis. A population-based study. Arthritis Rheum 1984; 27: 1353-61.

10 Cooper C, Wickham C. Rheumatoid arthritis, corticosteroid therapy and hip fracture. In: Christiansen C, Overgaard K,
eds. Osteoporosis 1990. Copenhagen: Osteopress, 1990: eds. Osteof

11 Michel B A, Bloch D A, Fries J F. Predictors of fracture in early rheumatoid arthritis. F Rheumatol 1991; 18: 804-8.

12 Hall G M, Hall M L, Ell P J, Perry L A, Doyle D V, Spector T D. Bone density (BMC) in postmenopausal rheumatoid arthritis (RA). Relationship with disease activity, steroid use and sex hormone levels [abstract]. Arthritis Rheum 1991; 34 (No 9 suppl): D89.

13 Ropes M W Bennet G A, Caleb S, Jacox R, Jesser R A Revision of diagnostic criteria for rheumatoid arthritis. Bull Rheum Dis 1958; 9: 175-6.

14 Ritchie D M, Boyle J A, McInnes J M, et al. Clinical studies with an articular index for the assessment of joint tenderness in patients with rheumatoid arthritis. $Q \mathcal{F}$ Med 1968 147: $393-406$

15 van der Heijde D M F M, van Riel P L C M, van de Putte L B A. Health status assessment in early rheumatoid arthritis. Evidence of sensitivity in a double blind trial. Scand I Rheumatol 1990; 19: 407-12.

16 Mazess R B, Wahner H M Nuclear medicine and densitometry. In: Riggs B L Melton III L J, eds Osteoporosis: etiology, diagnosis, and management. New York: Raven Press, 1988: 251-95.

17 van der Heijde D M F M, van't Hof M A, van Riel P L C M, et al. Judging disease activity in clinical practice in rheumatoid arthritis: first step in the development of a disease activity score. Ann Rheum Dis 1990; 49: 916-20.

18 van der Heijde D M F M, van't Hof M A, van Riel P L C M van Leeuwen M A, van Rijswijk M H, van de Putte L B A. A comparison of validity of single variables and composed indices for measuring disease activity in rheumatoid arthritis. Ann Rheum Dis 1992; 51: 177-81.

19 SAS Institute Inc. SAS/STAT User's Guide, Version 6. 4th ed Cary, NC: SAS Institute Inc. 1989.

20 Smith E L, Gilligan C. Physical activity effects on bone metabolism. Calcif Tissue Int 1991; 49 (suppl) S50-4.

21 Gerber N J. Can exercise prevent osteoporosis? $\mathrm{Br} f$ Rheumatol 1991; 30: 2-4

22 Arend W P, Dayer J-M. Cytokines and cytokine inhibitors or antagonists in rheumatoid arthritis. Arthritis Rheum 1990; 33: 305-15.

23 Peck W A, Woods W L. The cells of bone. In: Riggs B L and Melton L J III, eds. Osteoporosis: etiology, diagnosis and and Melton L J III, eds. Osteoporosis: etiology, diagnosi

24 Pacifici R, Rifas L, Teitelbaum S, et al. Spontaneous release of interleukin 1 from human blood monocytes reflects bone formation in idiopathic osteoporosis. Proc Natl Acad Sci USA 1987; 84: 4616-20.

25 Laan R F J M, van Riel P L C M, van de Putte L B A. Bone mass in patients with rheumatoid arthritis. Ann Rheum Dis 1992; 51: 826-32.

26 Hologic $Q D R 1000 \times$ ray bone densitometer. Operator's manual and user's guide. Waltham, MA: Horologic Inc. June 1991.

27 Sambrook P N, Ansell B M, Foster S, Gumpel I M, Hesp R, Reeve J. Bone turnover in early rheumatoid arthritis. 2 . Reeve J. Bone turnover in early rheumatoid arthritis.
Longitudinal bone studies. Ann Rheum Dis 1985; 44: Longit

28 Gough A, Lilley J, Ayre S, Sheeran T, Emery P. Axial bone loss in early rheumatoid arthritis-an association with disease activity [abstract]. Arthritis Rheum 1991; 34 (No 9 , suppl): D99. 